Copyright $@ 2010$ Institute of Electrical and electronics Engineers, Inc.

All Rights reserved.

Personal use of this material, including one hard copy reproduction, is permitted.

Permission to reprint, republish and/or distribute this material in whole or in part for any other purposes must be obtained from the IEEE.

For information on obtaining permission, send an e-mail message to stds-igr@ieee.org.

By choosing to view this document, you agree to all provisions of the copyright laws protecting it.

Individual documents posted on this site may carry slightly different copyright restrictions.

For specific document information, check the copyright notice at the beginning of each document. 


\title{
Industrial Process Model Integration using a Blackboard Model within a Pan Stage Decision Support System
}

\author{
R. Dodd ${ }^{1}$, A. Chiou ${ }^{1}$, X.Yu ${ }^{2}$ and R.Broadfoot ${ }^{3}$ \\ ${ }^{1}$ Central Queensland University, Rockhampton, Queensland, Australia \\ Email:r.dodd@cqu.edu.au,a.chiou@cqu.edu.au \\ ${ }^{2}$ RMIT University, Melbourne, Victoria, Australia \\ Email:x.yu@rmit.edu.au \\ ${ }^{3}$ Queensland University of Technology, Brisbane, Queensland, Australia \\ Email:r.broadfoot@qut.edu.au
}

\begin{abstract}
This paper describes the critical features for information sharing and exchange between industrial pan stage process models within a knowledge based supervisory support system (KBSSS), for pan stage operations in a sugar mill. The innovation outlined underpins the integration of the industrial process models working cooperatively with a blackboard system to provide system forecasting of future pan stage operating conditions.

The primary topic of this paper will be a description of the approach and how it supports information exchange during the forecast process undertaken by the pan stage industrial process models with a focus on: (1) design features, (2) implementation and (3) application to the prediction process.
\end{abstract}

\section{Introduction}

Raw sugar production from cane is a nominally continuous operation, with 120-168 hours of processing per week, extending over 20-25 weeks of the harvest season. The crystallisation section, often loosely referred to as the pan stage, is the most complex part of the factory process where there are several batch wise and continuous crystallisation steps taking place concurrently [1].

In current Australian practice, two operators are normally employed on the pan stage. There is considerable process interaction between the pan stage and centrifugal stage although management of the centrifugals is undertaken by different operators. The overall strategic management of the pan stage is quite difficult because of the very large number of process streams of varying compositions and crystal growth rate characteristics which must be managed [2]. Often the pan stage is managed in a sub-optimal manner because an overview of operations encompassing cane receival section, juice processing stations, the pan stage and centrifugal station of the sugar mill is not available.

The pressures on the Australian sugar industry to reduce the costs of sugar manufacture and increase the consistency of producing sugar of high quality require a smarter strategy for operation. Previous research [3] acknowledges that no conventional software engineering methods exist to provide an overall solution to this industrial problem due to its complexity, the wide variety of information sources required to be managed, overall management objectives, lack of adequate sugar mill crystallisation stage industrial process models and requirements for advisory strategies and supporting advice [4] to validate recommendations. Such wide and varied requirements are not easily managed and no such software based system for their unification currently exists to provide a solution.

Currently, there is no such supervisory control system for pan stage operations neither in the Australian sugar industry nor, as best as known to the collaborators, in the world sugar industry with literature only referring to a rudimentary advisory system for pan stage operations developed over three decades ago [5].

This paper is organised as follows. Section 2 presents an overview of the KBSSS industrial process models used within the system. Section 3 discusses an overview of the blackboard model and its major features. Section 4 presents the method of interaction between the industrial pan stage process models and the blackboard system. Section 5 presents results for the approach with Section 6 concluding the paper by 
presenting a discussion and conclusions. Acknowledgements are presented in Section 7.

\section{KBSSS Process Model Overview}

The pan stage is a complicated feed-forward and feed-back series of operations superimposed upon a series of batch and continuous processing operations. In order to forward predict future pan stage operating conditions, a sequence of process models to describe the overall process is necessary. A series of industrial process models $[6,7,8,9]$ collectively working together to describe the primary pan stage inputs and outputs are required along with actual models of the internal workings of the pan stage itself.

An overall encompassing view of the factory sections is currently not available and hence operators are not able to predict future pan stage loadings with any assistance from existing factory systems. Furthermore, prediction facilities are not available to determine the consequence of operators actions other than the actual forward estimates the operators intuitively carry out. The technique described in this paper is an approach to help solve this problem and make such facilities available.

The KBSSS uses intelligent systems technologies to provide a standardised approach for pan operations by integrating data from a variety of information sources from different sections of the sugar mill, along with dynamic process models of the pan stage and the collective knowledge and expertise of pan stage experts [5]. The overall system architecture and framework was presented in prior research $[4,10,11]$.

A core feature of this system is a predictive mechanism to determine future pan stage operating conditions with industrial process models describing the pan stage and its interaction with the other sections of the sugar factory. This predictive behavior is a key characteristic of dynamic process models of the pan stage expert system framework [10]. The pan stage industrial process models $[6,7,8,9]$ are tightly integrated into and work in tandem with the expert system rule base and are a core system technology.

\section{Blackboard Model Overview}

The blackboard system is an artificial intelligence solution founded on the blackboard architectural-based model. The initial development of the blackboard system approach is synonymous with the Hearsay-II speech understanding system [12] for which the original approach was developed and consequently showcased as a major artificial intelligence system technology to aid in the solution of complex problems.
Blackboard systems are recognised as a very flexible and powerful system technique for expert systems applications requiring dynamic control decision and applications that combine multiple technologies $[13,14,15,16]$. Furthermore, blackboard systems are recognised as having no equal for their powerful combination of capabilities. The benefits of the blackboard approach allows for information exchange between knowledge sources. Blackboard systems are a mature intelligent system technology with techniques available for ensuring their efficiency and flexibility [14] despite the relatively limited publicity and awareness afforded to them, even within the area of intelligent systems.

The use of a blackboard system is analogous to a team of experts solving a problem. Collectively the team of experts, known as "knowledge sources", work towards an overall problem solution. Due to the individual expertise of knowledge sources, they each work on providing solutions to smaller parts of the problem that are specific to their domain. Once an overall specification of the problem has been constructed, each knowledge source interacts with the blackboard when information specific to their domain becomes available. This contribution then allows other knowledge sources the chance to apply themselves. This incremental approach continues with each knowledge source adding portions of the overall solution until the original problem is finally solved. A control mechanism is implemented which dictates the order that the knowledge sources may interact with the blackboard system. This establishes an effective and coherent method of interaction, between the knowledge sources, in problem solving.

A blackboard system consists of three major components:

1. Knowledge sources. Knowledge sources are the expert modules that interact with the blackboard.

2. Blackboard. The blackboard is the shared working memory.

3. Control mechanism. The control mechanism is the component used for scheduling the reading and writing of knowledge sources with the blackboard. This scheduling is used to organise the knowledge sources interaction with the blackboard in an efficient manner.

These blackboard model components and their interaction, in relation to the KBSSS, are depicted in Figure 1 with the control mechanism scheduling the overall interactions of the knowledge sources with the blackboard.

The blackboard approach allows diversity in the problem solving approaches able to be used by the knowledge sources, since these sources are totally independent and do not interact. The blackboard 


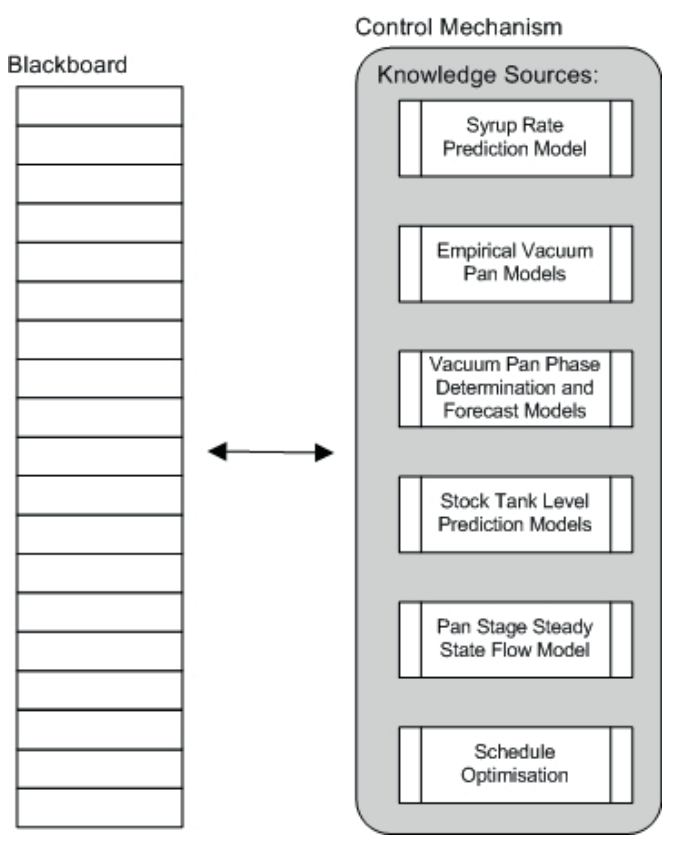

Figure 1. KBSSS blackboard system component interaction

architecture uses a common system for interaction between the blackboard and the knowledge sources. The blackboard can be viewed as a shared information repository of partial solutions, suggestions and contributed information that has been provided by knowledge sources.

The blackboard approach is a powerful problem solving architecture, allowing diversity amongst knowledge sources, providing a framework for combining fundamentally different knowledge sources and promotes modularity and independence during the design, implementation, testing and maintenance lifecycle phases of the application [13]. Given the requirements for an integration technique for the varying intelligent system technologies that form the basis for the major subsystems in the KBSSS design [4] the blackboard model is well suited to allow their integration and provide data exchange abilities.

\section{Interaction between Blackboard System and Industrial Process Models}

based upon the variety of knowledge sources used to carry out the overall forward prediction, the blackboard system is used to incrementally build a solution over the forecast period and work towards providing a prediction of future pan stage operating conditions. The blackboard system, as a shared working memory, stores results for each completed run of the pan stage process models and acts as the intermediary for the fuzzy inference engine to interact with for adaption of input parameters/model output to match against localized real-world conditions (the fuzzy inference engine processing and localization adaption is not covered in this paper).

At each prediction point the KBSSS builds a forward prediction and stores the value of each major process variable for each forecast point across the forecast period. This information is not only stored for information exchange between the subsystem models and access between the pan stage process models but also to allow review of archived data from previous forecasts. This is important to aid in performance reviews of the major system components through retrospective inspection.

Logically the blackboard storage structure representation appears in Figure 2. At each prediction point a series of data values are determined through use of the dynamic industrial process models working in tandem with the fuzzy rule base to generate a prediction of future pan stage operating conditions over the forecast period. The forecast period is broken down into a series of discrete predefined intervals over this forecast horizon and process variables tracked at each of these points. Successive predictions $p$ build up a series of prediction matrices consisting of variables var stored at each forecast interval $t$.

The blackboard system implementation uses an industry standard relational database to store the information from the KBSSS knowledge sources. Database SQL queries are used for the insertion and querying of information sources within the blackboard system.

Implementation-wise the underlying database uses the logical structure, presented in Figure 2, and maps this to a predefined database table structure. This compact representation allows flexible information storage for knowledge sources with varying forecast periods. This representation is suitable for use with the pan stage process model forecast process. The tuple representing a row in the blackboard database is:

\{KnowledgeSource, VariableName, ForecastTime, PredictionTime, VariableValue\}

where,

KnowledgeSource is the name of the knowledge source contributing the data,

VariableName is the name of the variable that data is being stored against, 


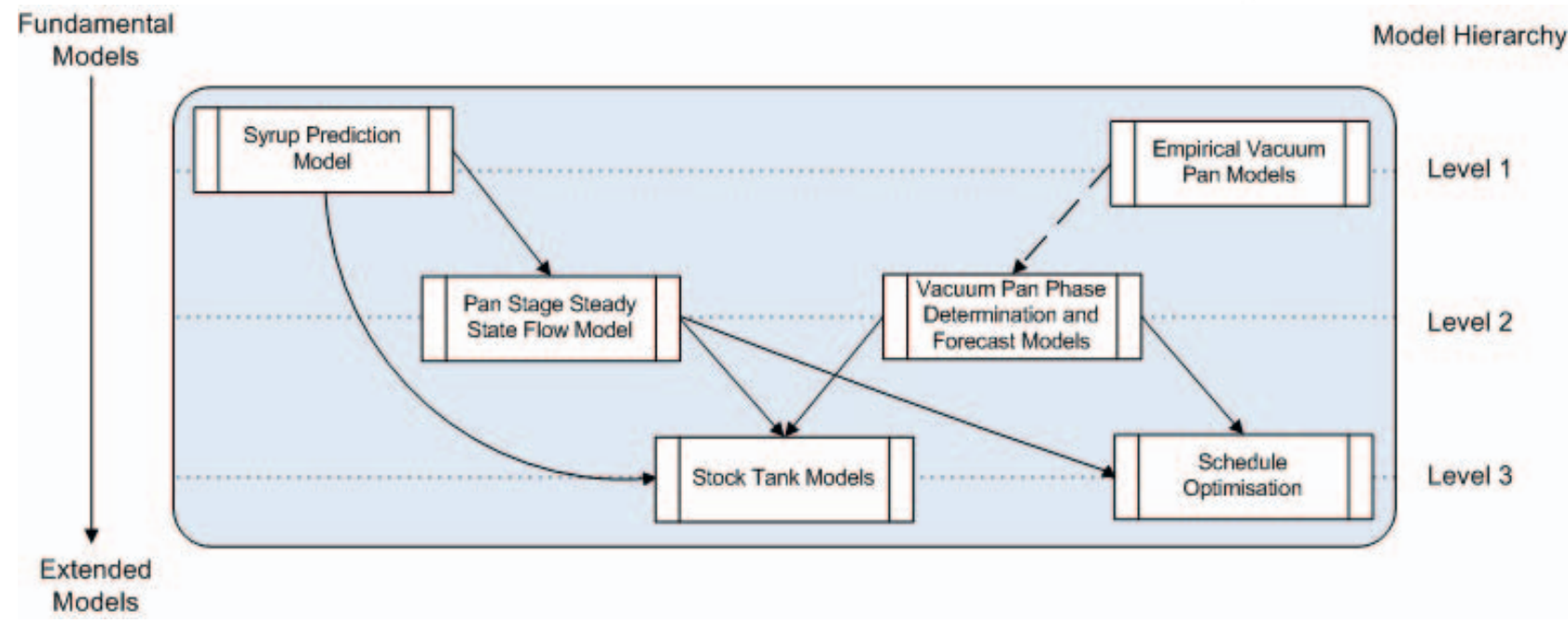

Figure 3. Model hierarchy for blackboard scheduling

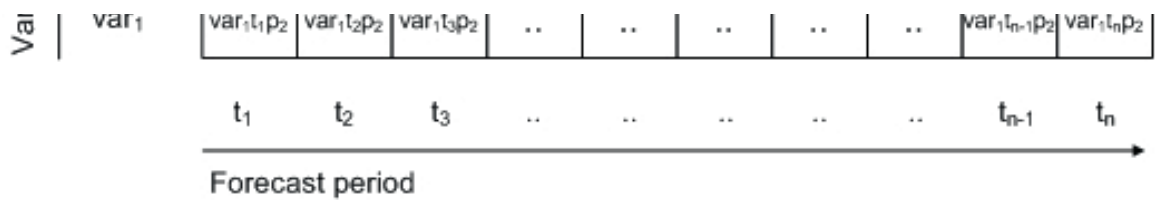

Figure 2. The blackboard system component implementation

ForecastTime is a date/time paired data value representing a forecast interval within the forecast period,

PredictionTime is a date/time paired data value representing the time that the forecast was generated; and

VariableValue is the actual data value stored.

An information hierarchy exists within the process models determining the order of their processing leading to a race condition in the scheduling of knowledge sources. Figure 3 shows the pan stage process model hierarchy. Pan stage process models higher in the hierarchy are dependent upon information from the more fundamental models at the lower levels. The arrows in the diagram display information flow required between the process models. The empirical vacuum pan models [6], as one of the essential and core pan stage process models, do not take part in scheduling by the blackboard scheduling system. The empirical vacuum pan models are static in nature and are used as local process model parameters for vacuum pan phase determination and forecast models. This relationship is in Figure 3 indicated by the broken arrow.

Models are iteratively processed based upon level within the defined hierarchy. Lower level models are scheduled for completion first with the next level of models in the hierarchy successively scheduled after their completion consecutively. In this respect, the control mechanism component of the blackboard model has been adapted from the traditional approach.

Scheduling of the procedures, for the individual pan stage process models, is undertaken by the blackboard control mechanism and maintains the data integrity through the ordered scheduling presented in Figure 3. The blackboard systems control mechanism is periodically run as part of the forecast process. This allows the forecast to harness new information on real world conditions and changes to sugar mill factory operational data from instrumentation.

\section{Results}

An example of the final published results for the blackboard model working to generate results of the syrup rate prediction model is provided in Table 1. A 90 minute forward forecast of sucrose and impurity quantities is presented for Racecourse sugar mill (Mackay, Australia) cane rake data at $11: 45 \mathrm{pm}$ on 03/09/2003 for information specific to the 2003 cane crushing season [8].

This forecast was undertaken using the syrup rate prediction model validated in prior research [10]. This 
model works in tandem with the dynamic allocation algorithm [8] to apportion predicted quantities to forecast intervals over the prediction horizon. These intervals form the basis of the prediction time that quantities are associated with.

Table 1 displays the results of the sucrose and impurity quantities forecast using the previously mentioned models to the blackboard information storage format. The VariableValue column stores the indicated quantity at the denoted forecast prediction times. The KnowledgeSource attribute from the tuple representation of the blackboard database is associated with the syrup rate prediction model in each row and has been omitted due to its common occurrence.

\section{Discussion and Conclusions}

This paper presents a blackboard framework, used as a fundamental supporting technology, of the KBSSS for pan stage operations within a sugar mill. The blackboard system shares common mechanics and properties of the traditional blackboard system approach with adaption of the scheduling component to harness the capabilities of pan stage process models in providing a future forecast of operating conditions. The blackboard model is used to facilitate information sharing between industrial pan stage process models during the forecast process.

The innovation outlined highlights how a blackboard model can be used for information sharing between industrial pan stage models to allow for data exchange. Results from fundamental process models are then available for use by extended process models in accordance with the hierarchy presented. The order in which the knowledge sources are called is determined by the control mechanism in accordance with the data dependencies that exist between process models.

The modification to the traditional blackboard model is in providing an ordering of tasks for the control mechanism to undertake, in order to avoid a race condition. However this series of tasks is not a strict linear interaction with only the blackboard system. Fuzzy pre-adjustment of process model input parameters and post-adjustment of process model outputs (not covered in this paper) also occurs. This modification results in a hybrid control mechanism that is well suited to the blackboard model working in tandem with the pan stage process models as part of the overall KBSSS design.

The method presented in this paper when working in tandem with the syrup prediction model allows an overview of syrup loadings to the pan stage by relating the cane receival and juice processing sections of the factory directly to the pan stage. Results from this process model are then shared through the blackboard system, as illustrated in the paper, to allow extended industrial process models to collaboratively work in building a future forecast of pan stage operating conditions.

\section{Acknowledgements}

The project contributors would like to thank the staff at Racecourse Sugar Mill, Mackay, Australia for their cooperation and support. This project was supported by an Australian Research Council linkage grant. The funding assistance provided by the Sugar Research Institute, Brisbane, Australia is also acknowledged and appreciated.

\section{References}

[1] R. Broadfoot and A. Beath, "Modelling of batch sugar centrifugal performance", in Proceedings of 26th Australasian Chemical Engineering Conference, 1988, pp.294-303.

[2] K. Miller and R. Broadfoot. "Crystal growth rates in high grade massecuite boilings", in Proceedings of the Australian Society of Sugar Cane Technologists Conference, 1997, pp.441-447.

[3] R. Dodd, "A knowledge based supervisory support system for pan stage operations in a sugar mill", Ph.D dissertation, Royal Melbourne Institute of Technology University, Melbourne, Victoria, Australia, 2009.

Table 1. Blackboard Results for Sucrose and Impurity Quantities (tonnes) for the Syrup Rate Prediction Model Knowledge Source

\begin{tabular}{|l|l|l|r|}
\hline VariableName & ForecastTime & PredictionTime & VariableValue \\
\hline SucroseQuantity & $11: 45 \mathrm{PM}$ & $12: 00 \mathrm{AM}$ & 18.22 \\
SucroseQuantity & $11: 45 \mathrm{PM}$ & $12: 15 \mathrm{AM}$ & 16.78 \\
SucroseQuantity & $11: 45 \mathrm{PM}$ & $12: 30 \mathrm{AM}$ & 19.73 \\
SucroseQuantity & $11: 45 \mathrm{PM}$ & $12: 45 \mathrm{AM}$ & 18.71 \\
SucroseQuantity & $11: 45 \mathrm{PM}$ & $1: 00 \mathrm{AM}$ & 19.19 \\
SucroseQuantity & $11: 45 \mathrm{PM}$ & $1: 15 \mathrm{AM}$ & 19.07 \\
ImpurityQuantity & $11: 45 \mathrm{PM}$ & $12: 00 \mathrm{AM}$ & 2.25 \\
ImpurityQuantity & $11: 45 \mathrm{PM}$ & $12: 15 \mathrm{AM}$ & 2.08 \\
ImpurityQuantity & $11: 45 \mathrm{PM}$ & $12: 30 \mathrm{AM}$ & 2.44 \\
ImpurityQuantity & $11: 45 \mathrm{PM}$ & $12: 45 \mathrm{AM}$ & 2.48 \\
ImpurityQuantity & $11: 45 \mathrm{PM}$ & $1: 00 \mathrm{AM}$ & 2.37 \\
ImpurityQuantity & $11: 45 \mathrm{PM}$ & $1: 15 \mathrm{AM}$ & 2.36 \\
\hline
\end{tabular}


[4] R. Dodd, A. Chiou, X. Yu and R. Broadfoot. "Framework for a smart supervisory control system for a sugar mill crystallisation stage", in Proceedings of the 6th IEEE International Conference of Industrial Informatics, 2008, pp. 463-468.

[5] J. Frew and P. Wright, "Sugar crystallisation: a pan stage advisory scheme", in Proceedings of Queensland Society of Sugar Cane Technologists, 1976, pp. $191-198$.

[6] R. Dodd, R. Broadfoot, X. Yu and A. Chiou, "Empirical pan modelling of vacuum pans for a sugar mill crystallization stage", in Proceedings of Australian Society of Sugar Cane Technologists, 2005, pp.423-436.

[7] R. Dodd, R. Broadfoot, X. Yu and A. Chiou, "Process models for a sugar mill crystallisation stage knowledge based supervisory support system", in Proceedings of I*PROMS Virtual International Conference, 2008, pp. 1-6.

[8] R. Dodd, R. Broadfoot, A. Chiou, and X. Yu, "Dynamic allocation of predicted quantities to forecast intervals for pan stage supervisory support system process models", in Proceedings of the 2009 IEEE International Conference on Industrial Technology, 2009, pp. 1-6.

[9] R. Dodd, R. Broadfoot, A. Chiou, and X. Yu, "Pan stage steady state flow model for integration within a knowledge based supervisory support system", in Proceedings of the 2009 IEEE International Conference on Industrial Technology, 2009, pp. 1-6.

[10] R. Dodd, R. Broadfoot, X. Yu and A. Chiou, "'Development of smart supervisory control system in a sugar mill crystallisation stage", in Proceedings of I*PROMS Virtual International Conference, 2005, pp.527-534.

[11] X. Yu, A. Chiou and R. Dodd, "A novel decision support framework for industrial processes", in Proceedings of the 31 st Annual Conference of IEEE Industrial Electronics Society, 2005, pp. 2210-2215.

[12] L. Erman, F. Hayes-Roth, V. Lesser and D. Raj Reddy, "The hearsay-II speech-understanding system: integrating knowledge to resolve uncertainty", Computing Surveys, vol. 12, no. 2, pp. 213-253, June 1980.

[13] D. Corkill, "Blackboard systems", AI Expert, vol. 6, no. 9, pp. 40-47, September 1991.

[14] D. Corkill, Q. Gallagher and P. Johnson, "Achieving flexibility, efficiency, and generality in blackboard architectures", in Proceedings of National Conference on Artificial Intelligence, 1987, pp. 18-23.

[15] N. Carver, "A revisionist view of blackboard systems", in Proceedings of the 1997 Midwest Artificial Intelligence and Cognitive Science Society Conference, 1997.
[16] D. Corkill, "Countdown to success: dynamic objects, gbb, and radarsat-1", Communications of the ACM, vol. 40, no. 5, pp. 48-58, May 1997. 